\title{
Numerical Method for Three-Dimensional Heat Conduction in Cylindrical and Spherical Coordinates
}

\author{
Xinxin Jia, Zongrui Hao, Hu Wang, Lei Wang, Xin Wang \\ Instrumentation of Shandong Academy of Sciences, Qingdao 266061, China \\ upcjxx@163.com
}

\begin{abstract}
According to the differential equations of heat conduction on cylindrical and spherical coordinate system, numerical solution of the discrete formula on cylindrical and spherical coordinate system with high accuracy were derived. Compared with the analytical solution, this discrete formula was verified with a high degree of accuracy. To make the complex dispersion coefficient of diffusion term $\theta$ more concrete in spherical coordinates, this paper derived the discretion coefficient of diffusion term $\theta$ by the first mean value theorem of integral. The accurate schemes provide a good reference for researchers whose work in solving the equation of heat conduction of three-dimensional cylindrical coordinates and spherical coordinates, and it will provide accurate numerical schemes and the theoretical basis for solving practical engineering problems.
\end{abstract}

Keywords: Differential equation of heat conduction; Spherical coordinate; Numerical heat transfer; the first mean value theorem for integral.

\section{Introduction}

Numerical Heat Transfer has been widely used to solve practical complex heat transfer calculations [1-3]. The important thing to solve the problem was how to discrete the thermal conductivity differential equations. Numerical Heat Transfer in two-dimensional cylindrical coordinates and polar coordinates equation of heat conduction were applied widely. When referring to the discretion schemes of three-dimensional cylindrical and spherical coordinates, now there is not a relatively discrete format for it $[4,5]$.

At the present, when it comes to the questions of cylinder and sphere in numerical heat transfer, it is just simplified into the radial or two-dimensional polar coordinates, which caused a lot of inconvenience in computation, promotion and application. Therefore, it is very important for us to solve to the three-dimensional cylinder coordinates thermal conductivity partial differential equations and thermal conductivity of the sphere of partial differential equations. This paper derived high precision cylindrical coordinates and spherical coordinate's equation of heat conduction discrete schemes and compared with one-dimensional analytical solution to verify the accuracy of the numerical solution.

\section{Problem description}

The partial differential equations for heat conduction was established in the Cartesian coordinate system and the energy principle and Fourier's [6] law was used. Then we will have thermal conductivity of partial differential Eq. (1) as follows:

$$
\frac{\partial}{\partial \tau}(\rho c T)=\lambda \frac{\partial^{2} T}{\partial x^{2}}+\lambda \frac{\partial^{2} T}{\partial y^{2}}+\lambda \frac{\partial^{2} T}{\partial z^{2}}+S
$$

Where $\lambda$ is thermal conductivity, $c$ is the heat capacity of the thermal conductivity, $S$ is inner heat source.

The cylindrical coordinate system Eq.(2) and the spherical coordinate system Eq.(3) of the differential equations[6] are described as follows:

$$
\frac{\partial}{\partial \tau}(\rho c T)=\frac{1}{r} \frac{\partial}{\partial r}\left(\lambda r \frac{\partial T}{\partial r}\right)+\frac{1}{r^{2}} \frac{\partial}{\partial \varphi}\left(\lambda \frac{\partial T}{\partial \varphi}\right)+\frac{\partial}{\partial z}\left(\lambda \frac{\partial T}{\partial z}\right)+S, \quad 0 \leq \varphi \leq 2 \pi
$$




$$
\begin{gathered}
\frac{\partial}{\partial \tau}(\rho c T)=\frac{1}{r^{2}} \frac{\partial}{\partial r}\left(\lambda r^{2} \frac{\partial T}{\partial r}\right)+\frac{1}{r^{2} \sin ^{2} \theta} \frac{\partial}{\partial \varphi}\left(\lambda \frac{\partial T}{\partial \varphi}\right)+\frac{1}{r^{2} \sin \theta} \frac{\partial}{\partial \theta}\left(\lambda \sin \theta \frac{\partial T}{\partial \theta}\right)+S \\
0 \leq \theta \leq \pi, 0 \leq \varphi \leq 2 \pi
\end{gathered}
$$

In general, there are two methods to discrete the equation of heat conduction, that is, Taylor series expansion method and finite volume method. In order to ensure the physical significance [7, 8], this paper will derive the thermal conductivity of partial differential equations by finite volume method.

\section{Literature References}

First, multiply $r$ on both sides of the Eq.(2), then the integral equation in control volume and unsteady time items are shown in Figure1. The Eq.(2) changed into Eq.(4) as follows:

$$
\begin{aligned}
& \int_{t}^{t+\Delta t} \iiint_{V} r \frac{\partial}{\partial \tau}(\rho c T) d r d d z=\int_{t}^{t+\Delta t} \iiint_{V}\left[\frac{\partial}{\partial r}\left(\lambda r \frac{\partial T}{\partial r}\right)+\frac{1}{r} \frac{\partial}{\partial}\left(\lambda \frac{\partial T}{\partial}\right)+r \frac{\partial}{\partial z}\left(\lambda \frac{\partial T}{\partial z}\right)\right] d r d d z \\
& +\iint_{t}^{t+\Delta t} \iint_{V} r S d r d d z
\end{aligned}
$$

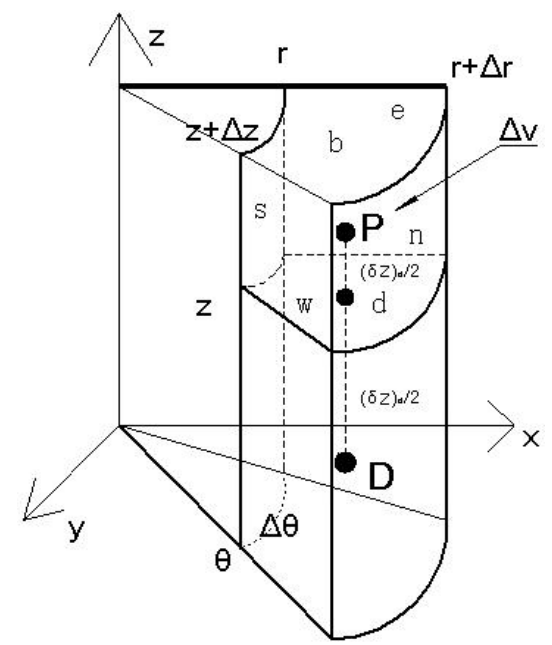

Fig.1 Cylindrical coordinates control volume

\subsection{The integral of the unsteady terms}

After integrating we can gain the unsteady term in the following forms:

$$
\int_{t}^{t+\Delta t} \int_{s}^{n} \int_{w}^{e} \int_{b}^{d} r \frac{\partial T}{\partial \tau} d r d d z=\frac{\left(r_{n}+r_{s}\right) \Delta r}{2} \Delta \Delta z\left(T_{P}-T_{P}^{0}\right)
$$

\subsection{The integral of the diffusion terms $r, \varphi, z$}

After integrating the diffusion terms into the following forms:

$$
\begin{aligned}
& \int_{t}^{t+\Delta t} \int_{s}^{n} \int_{w}^{e} \int_{w}^{d} \frac{\partial}{\partial r}\left(\lambda r \frac{\partial T}{\partial r}\right) d r d \varphi d z d t=\Delta \varphi \Delta z \Delta t \int_{s}^{n} \frac{\partial}{\partial r}\left(\lambda r \frac{\partial T}{\partial r}\right) d r=\Delta \varphi \Delta z \Delta t\left[\lambda_{n} r_{n} \frac{T_{N}-T_{P}}{(\delta r)_{n}}-\lambda_{s} r_{s} \frac{T_{P}-T_{S}}{(\delta r)_{s}}\right] \\
& \int_{t}^{t+\Delta t} \int_{s}^{n} \int_{w}^{e} \int_{b}^{d} \frac{1}{r} \frac{\partial}{\partial}\left(\lambda \frac{\partial T}{\partial}\right) d r d d z d t=\ln \left(\frac{r_{n}}{r_{s}}\right) \Delta z \Delta t \int_{w}^{e} \frac{\partial}{\partial}\left(\lambda \frac{\partial T}{\partial}\right) d=\ln \left(\frac{r_{n}}{r_{s}}\right) \Delta z \Delta t\left[\lambda_{e} \frac{T_{E}-T_{P}}{(\delta)_{e}}-\lambda_{w} \frac{T_{P}-T_{W}}{(\delta)_{w}}\right] \\
& \int_{t}^{t+\Delta t} \int_{s}^{n} \int_{w}^{b} \int_{w}^{b} r \frac{\partial}{\partial z}\left(\lambda \frac{\partial T}{\partial z}\right) d r d d z d t=\frac{\left(r_{n}+r_{s}\right) \Delta r}{2} \Delta \Delta t \int_{d}^{b} \frac{\partial}{\partial z}\left(\lambda \frac{\partial T}{\partial z}\right) d z \\
& =\frac{\left(r_{n}+r_{s}\right) \Delta r}{2} \Delta \Delta t\left[\lambda_{b} \frac{T_{B}-T_{P}}{(\delta z)_{b}}-\lambda_{d} \frac{T_{P}-T_{D}}{(\delta z)_{d}}\right]
\end{aligned}
$$

\subsection{The integral of the source term}

$$
\int_{t}^{t+\Delta t} \int_{s}^{n} \int_{w}^{e} \int_{b}^{d} r S d r d \varphi d z d t=\frac{\left(r_{n}+r_{s}\right) \Delta r}{2} \Delta \varphi \Delta z \Delta t\left(S_{C}+S_{P} T_{P}\right)
$$

Here, the discrete equations will be solved by the techniques for linear equation, it is sufficient to express the average value $S$ as:

$$
S=S_{C}+S_{P} T_{P}
$$


where, $S_{C}$ stands for the constant part of $S, S_{P}$ is the coefficient of $T_{P}$.

\subsection{The discrete Equation for cylinder}

With the linearized source expression, the discrete equation would changed into the Eq.(4) as having the form:

$$
a_{P} T_{P}=a_{E} T_{E}+a_{W} T_{W}+a_{N} T_{N}+a_{S} T_{S}+a_{B} T_{B}+a_{D} T_{D}+b
$$

Where:

$$
\begin{aligned}
& a_{E}=\frac{\ln \left(\frac{r_{n}}{r_{s}}\right) \Delta z}{(\delta \varphi)_{e} / \lambda_{e}}, a_{W}=\frac{\ln \left(\frac{r_{n}}{r_{s}}\right) \Delta z}{(\delta \varphi)_{w} / \lambda_{w}}, a_{N}=\frac{r_{n} \Delta \varphi \Delta z}{(\delta r)_{n} / \lambda_{n}}, a_{S}=\frac{r_{s} \Delta \varphi \Delta z}{(\delta r)_{s} / \lambda_{s}}, \\
& a_{B}=\frac{\left(r_{n}+r_{s}\right) \Delta r \Delta}{2(\delta z)_{b} / \lambda_{b}}, a_{D}=\frac{\left(r_{n}+r_{s}\right) \Delta r \Delta}{2(\delta z)_{d} / \lambda_{d}}, b=S_{C} \Delta V+a_{P}^{0} T_{P}^{0} \\
& a_{P}=a_{E}+a_{W}+a_{N}+a_{S}+a_{B}+a_{D}+a_{P}^{0}-S_{P} \Delta V, \Delta V=\frac{\left(r_{n}+r_{s}\right) \Delta r}{2} \Delta \Delta z, \\
& a_{P}^{0}=\frac{(\rho c)_{P} \Delta V}{\Delta t} .
\end{aligned}
$$

At this point, it is interesting to examine the physical significance of the various coefficients in the discrete equation. The neighbor coefficients $a_{E}, a_{W}, a_{N}, a_{S}, a_{E}, a_{D}$ represent the conductance between the point $\boldsymbol{P}$ and the corresponding neighbors. The term $a_{P}^{0} T_{P}^{0}$ is the internal energy contained in the control volume at time $\boldsymbol{t}$. The constant term $\boldsymbol{b}$ consists of this internal energy and the rate of heat generation in the control volume resulting from $S_{C}$. The $\Delta V$ is the volume of the control volume. The center-point coefficient $a_{P}$ is the sum of all neighbor coefficients and contains a contribution from the linearized source term.

\section{The discrete control equation of spherical coordinates}

At this stage, multiply $r^{2} \sin ^{2} \theta$ on both sides of the Eq.(2), then the integral equation that was in control volume and unsteady time items points are shown in Figure 2. The new equation is as follows:

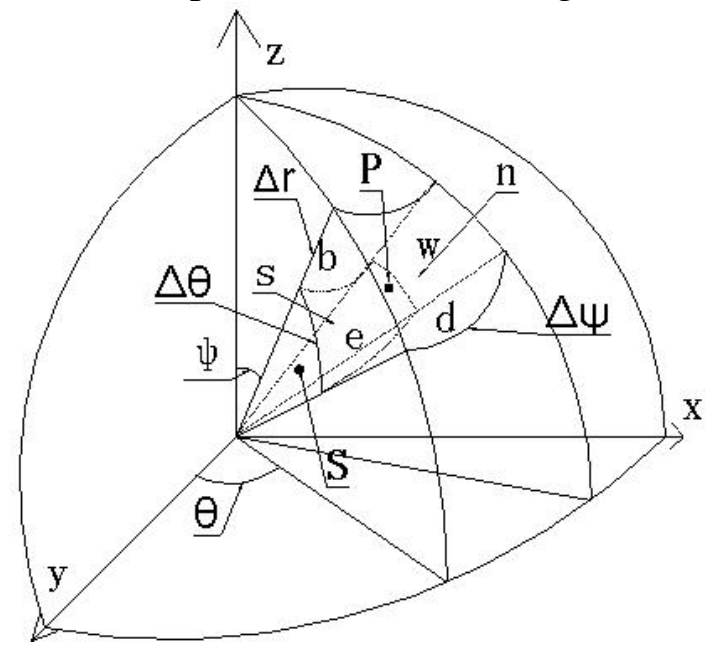

Fig.2 Spherical coordinate control volume

$$
\begin{aligned}
& \int_{t}^{t+\Delta t} \iiint_{V} r^{2} \sin ^{2} \theta \frac{\partial}{\partial \tau}(\rho c T) d t d r d \theta d=\int_{t}^{t+\Delta t} \iiint_{V}\left[\sin ^{2} \theta \frac{\partial}{\partial r}\left(\lambda r^{2} \frac{\partial T}{\partial r}\right)+\frac{\partial}{\partial}\left(\lambda \frac{\partial T}{\partial}\right)\right. \\
& \left.+\sin \theta \frac{\partial}{\partial \theta}\left(\lambda \sin \theta \frac{\partial T}{\partial \theta}\right)\right] d t d r d \theta d+\int_{t}^{t+\Delta t} \iiint_{V} S \cdot r^{2} \sin ^{2} \theta d t d r d \theta d
\end{aligned}
$$

\subsection{The integral of the unsteady terms}

After integrating the unsteady term into the following forms:

$\int_{t}^{t+\Delta t} \int_{s}^{n} \int_{w}^{e} \int_{b}^{d} r^{2} \sin ^{2} \theta \frac{\partial}{\partial \tau}(\rho c T) d t d r d \theta d \varphi=\frac{\left(r_{n}^{3}-r_{s}^{3}\right)}{6}\left(\Delta \theta+\sin \theta_{w} \cos \theta_{w}-\sin \theta_{e} \cos \theta_{e}\right) \Delta \varphi\left(T_{P}-T_{P}^{0}\right)$ 


\subsection{The integral of the diffusion terms $r, \varphi$}

After integrating the diffusion term $r, \varphi$ becomes the following forms:

$$
\begin{aligned}
& \int_{t}^{t+\Delta t} \int_{s}^{t} \int_{w}^{e} \int_{b}^{d} \sin ^{2} \theta \frac{\partial}{\partial r}\left(\lambda r^{2} \frac{\partial T}{\partial r}\right) d t d r d \theta d \varphi=\frac{1}{2}\left(\Delta \theta+\sin \theta_{w} \cos \theta_{w}-\sin \theta_{e} \cos \theta_{e}\right) \Delta \varphi \Delta t\left[r_{n}^{2} \frac{T_{N}-T_{P}}{(\delta r)_{n}}-r_{s}^{2} \frac{T_{P}-T_{S}}{(\delta r)_{s}}\right] \\
& \int_{t}^{t+\Delta t} \int_{s}^{n} \int_{w}^{e} \int_{b}^{d} \frac{\partial}{\partial}\left(\lambda \frac{\partial T}{\partial}\right) d t d r d \theta d=\left[\frac{T_{B}-T_{P}}{(\delta)_{b}}-\frac{T_{P}-T_{D}}{(\delta)_{d}}\right] \Delta \theta \Delta r \Delta t
\end{aligned}
$$

\subsection{Application integral mean value theorem derive the diffusion term $\theta$}

In the derivation of the integral term, this paper use the integral mean value theorem which will derive the diffusion term $\theta$ scientifically and reasonably.

Obviously, the heat flux that leaves one control volume through a particular face must be identical to the flux that enters the next control volume through the same face. Otherwise, the overall balance would not be satisfied. It is the fact that the energy across the interface $\theta_{e}$ to $\theta_{w}$ continuously, so the heat conduction equation can be thought as a continuity equation. Because of the diffusion $\operatorname{term} \theta \in[0, \pi]$, it happens for $\forall \theta \in[0, \pi]$, and we have $\sin \theta \geq 0$. Based on the above two conditions of continuity and $\sin \theta \geq 0$, which satisfied the integral mean value theorem[9], the diffusion term $\theta$ and Eq.(3) are changed as the following $\exists \xi \in[w, e]$ shows, so that the $\theta$ conduction term denotes:

$$
\int_{t}^{t+\Delta t} \int_{s}^{t} \int_{w}^{e} \int_{b}^{d} \sin \theta \frac{\partial}{\partial \theta}\left(\lambda \sin \theta \frac{\partial T}{\partial \theta}\right) d t d r d \theta d \varphi=\sin \theta_{\xi}^{t+\Delta t} \int_{t}^{t} \int_{s}^{n} \int_{w}^{e} \int_{b}^{d} \frac{\partial}{\partial \theta}\left(\lambda \sin \theta \frac{\partial T}{\partial \theta}\right) d t d r d \theta d \varphi
$$

It can be seen that when $\Delta \theta$ is much smaller, the value $\theta_{\xi} \approx \theta_{P}$.

$$
\begin{aligned}
& \sin \theta_{\xi}^{t+\Delta t} \int_{t}^{t} \int_{s}^{n} \int_{w}^{e} \int_{b}^{d} \frac{\partial}{\partial \theta}\left(\lambda \sin \theta \frac{\partial T}{\partial \theta}\right) d t d r d \theta d \approx \sin \theta_{P} \int_{t}^{t+\Delta t} \int_{s}^{n} \int_{w}^{e} \int_{w}^{d} \frac{\partial}{\partial \theta}\left(\lambda \sin \theta \frac{\partial T}{\partial \theta}\right) d t d r d \theta d \\
& =\sin \theta_{P}\left[\sin \theta_{e} \frac{T_{E}-T_{P}}{(\delta \theta)_{e}}-\sin \theta_{w} \frac{T_{P}-T_{W}}{(\delta \theta)_{w}}\right] \Delta \Delta r \Delta t
\end{aligned}
$$

\subsection{The integral of the source term}

After integrating the source term $S$ becomes the following forms:

$$
\int_{t}^{t+\Delta t} \int_{s}^{n} \int_{w}^{e} \int_{b}^{d} r^{2} \sin ^{2} \theta \cdot S d t d r d \theta d \varphi=\frac{\left(r_{n}^{3}-r_{s}^{3}\right)}{6}\left(\Delta \theta+\sin \theta_{w} \cos \theta_{w}-\sin \theta_{e} \cos \theta_{e}\right) \Delta \varphi \Delta t\left(S_{C}+S_{P} T_{P}\right)
$$

\subsection{The discrete Equation for sphere}

The discrete equation can easily be seen as follows:

$$
a_{P} T_{P}=a_{E} T_{E}+a_{W} T_{W}+a_{N} T_{N}+a_{S} T_{S}+a_{B} T_{B}+a_{D} T_{D}+b
$$

Where

$$
\begin{aligned}
& a_{E}=\frac{\Delta \varphi \Delta r \sin \theta_{P} \sin \theta_{e}}{(\delta \theta)_{e} / \lambda}, a_{W}=\frac{\Delta \varphi \Delta r \sin \theta_{P} \sin \theta_{w}}{(\delta \theta)_{w} / \lambda}, \\
& a_{N}=\frac{\left(\Delta \theta+\sin \theta_{w} \cos \theta_{w}-\sin \theta_{e} \cos \theta_{e}\right) \Delta \cdot r_{n}^{2}}{2(\delta r)_{n} / \lambda}, \\
& a_{S}=\frac{\left(\Delta \theta+\sin \theta_{w} \cos \theta_{w}-\sin \theta_{e} \cos \theta_{e}\right) \Delta \varphi \cdot r_{s}^{2}}{2(\delta r)_{s} / \lambda}, \\
& a_{B}=\frac{\Delta \theta \Delta r}{(\delta)_{b} / \lambda_{b}}, a_{D}=\frac{\Delta \theta \Delta r}{(\delta \varphi)_{d} / \lambda_{d}}, b=S_{C} \Delta V+a_{P}^{0} T_{P}^{0}, \\
& a_{P}=a_{E}+a_{W}+a_{N}+a_{S}+a_{B}+a_{D}+a_{P}^{0}-S_{P} \Delta V, \\
& \Delta V=\frac{\left(r_{n}^{3}-r_{s}^{3}\right)}{6}\left(\Delta \theta+\sin \theta_{w} \cos \theta_{w}-\sin \theta_{e} \cos \theta_{e}\right), \\
& a_{P}^{0}=\frac{(\rho c)_{P} \Delta V}{\Delta t}, 0 \leq \theta \leq \pi, 0 \leq \leq 2 \pi .
\end{aligned}
$$

With the different express, the terms of $a_{E}, a_{W}, a_{N}, a_{S}, a_{B}, a_{D}, P, a_{P}^{0} T_{P}^{0}$ etc. are the same as the part 3 shown. 


\section{Numerical value comparison}

\subsection{Application of discrete equations and grid independent analysis}

To illustrate the workings of the discrete equations this paper gives a detailed example of cylindrical heat conduction. The inner and outer diameter of the cylinder were $R_{1}$ and $R_{2}$ The temperature of the inside and outside of the cylinder was $T_{1}$ and $T_{2}$ and the radial heat conduction analytical solution is as follows:

$$
T=T_{1}+\left(T_{2}-T_{1}\right) \frac{\ln \left(R / R_{1}\right)}{\ln \left(R_{2} / R_{1}\right)}
$$

We consider it as a steady and three-dimensional cylinder, and the heat constant cylindrical boundary conditions (the problem data) are as follows:

$$
\left\{\begin{array}{l}
\frac{1}{r} \frac{\partial}{\partial r}\left(\lambda r \frac{\partial T}{\partial r}\right)+\frac{1}{r^{2}} \frac{\partial}{\partial}\left(\lambda \frac{\partial T}{\partial}\right)+\frac{\partial}{\partial z}\left(\lambda \frac{\partial T}{\partial z}\right)=0 \\
R_{1}=1, T_{1}=1 \\
R_{2}=2, T_{2}=1000 \\
0 \leq \leq 2 \pi
\end{array}\right.
$$

The numerical computed in this paper as well as the analytical solutions are compared in Figure3. Given the coarseness of the triangle that numerical solution gives reasonable agreement with the analytical solution, the error between the numerical and analytical solution was $8.86 \times 10^{-3} \%$.

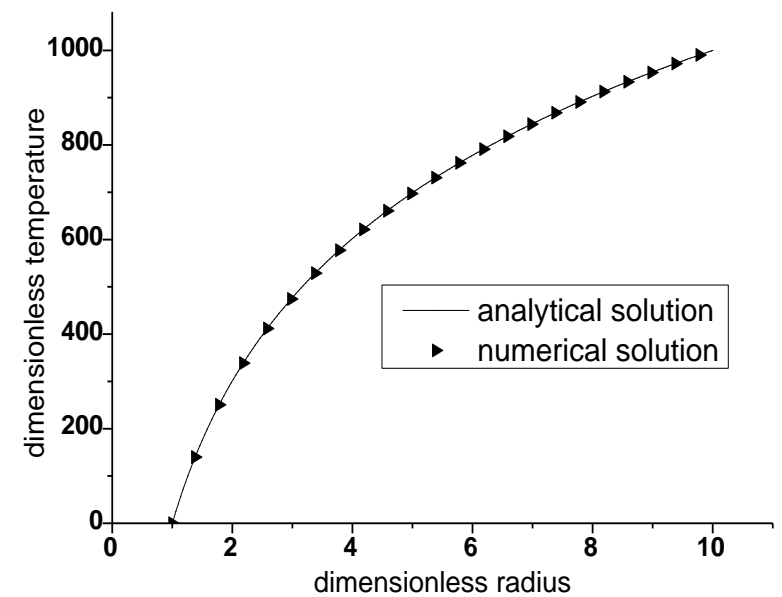

Fig.3 Analytical solution and numerical solution

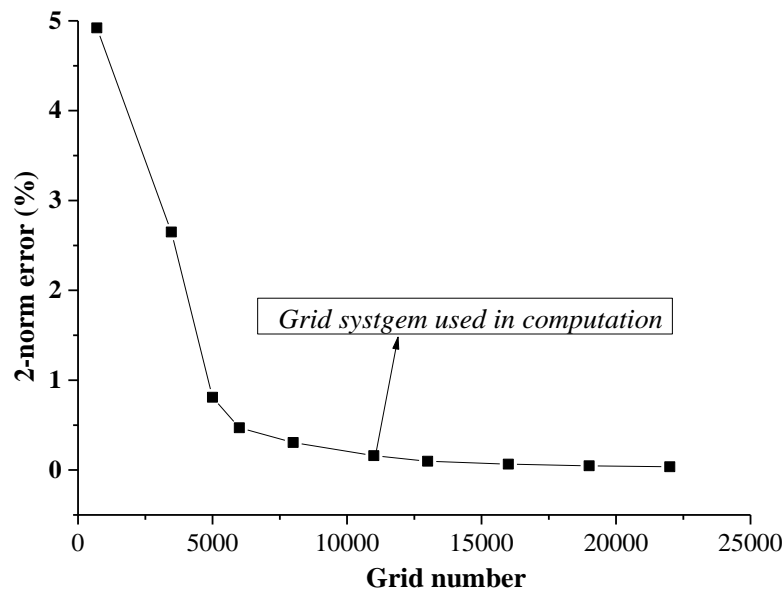

Fig.4 Grids number and error relations

To quantify the accuracy of the reconstruction formulas between numerical solution and analytical solution we define the relative error as:

$$
\text { error }=\frac{\left\|T-T_{\text {num }}\right\|_{2}}{\|T\|_{2}} \times 100 \%
$$

Where the error means the error between the solutions of the governing equations, and $\boldsymbol{T}$ refers to the solutions by means of the analytical solution; $\boldsymbol{T}_{\text {num }}$ refers to the solutions by means of the numerical solution.

The error means $L_{2}$-norm [10]: $\|T\|_{2}=\left(\sum_{i=1}^{n} x_{i}{ }^{2}\right)^{\frac{1}{2}}$

Based on the error obtained from grid-independent solution [11], the relation between the equation error and grid number is shown in Figure 4. When the grid number reaching 5000, the calculation error control is less than $1 \%$; with the encryption of the grid, the calculation error is reduced gradually. The analytical solution error is $8.86 \times 10^{-3} \%$, the choice of computational grid number is $37 \times 20 \times 16=11840$. 


\subsection{The validation of the thermal equation of the sphere}

To illustrate the workings of the sphere equations, this paper gives a detailed example of sphere heat conduction. The inner and outer diameter were $R_{1}$ and $R_{2}$. The temperature of the inside and outside of the sphere was $\boldsymbol{T}_{1}$ and $\boldsymbol{T}_{2}$.

We consider it as a steady and three-dimensional sphere, and the heat constant boundary conditions (the problem data) are as follows:

$$
\left\{\begin{array}{l}
\frac{1}{r^{2}} \frac{\partial}{\partial r}\left(\lambda r^{2} \frac{\partial T}{\partial r}\right)+\frac{1}{r^{2} \sin ^{2} \theta} \frac{\partial}{\partial}\left(\lambda \frac{\partial T}{\partial}\right)+\frac{1}{r^{2} \sin \theta} \frac{\partial}{\partial \theta}\left(\lambda \sin \theta \frac{\partial T}{\partial \theta}\right)=0 \\
R_{1}=1, T_{1}=1 \\
R_{2}=2, T_{2}=100 \\
0 \leq \leq 2 \pi, 0 \leq \theta \leq \pi
\end{array}\right.
$$

The numerical computed in this paper as well as analytical solutions are compared in Figure5. Given the coarseness of the triangle that numerical solution gives reasonable agreement with the analytical solution, the error between the numerical and analytical solution was $0.53 \%$.
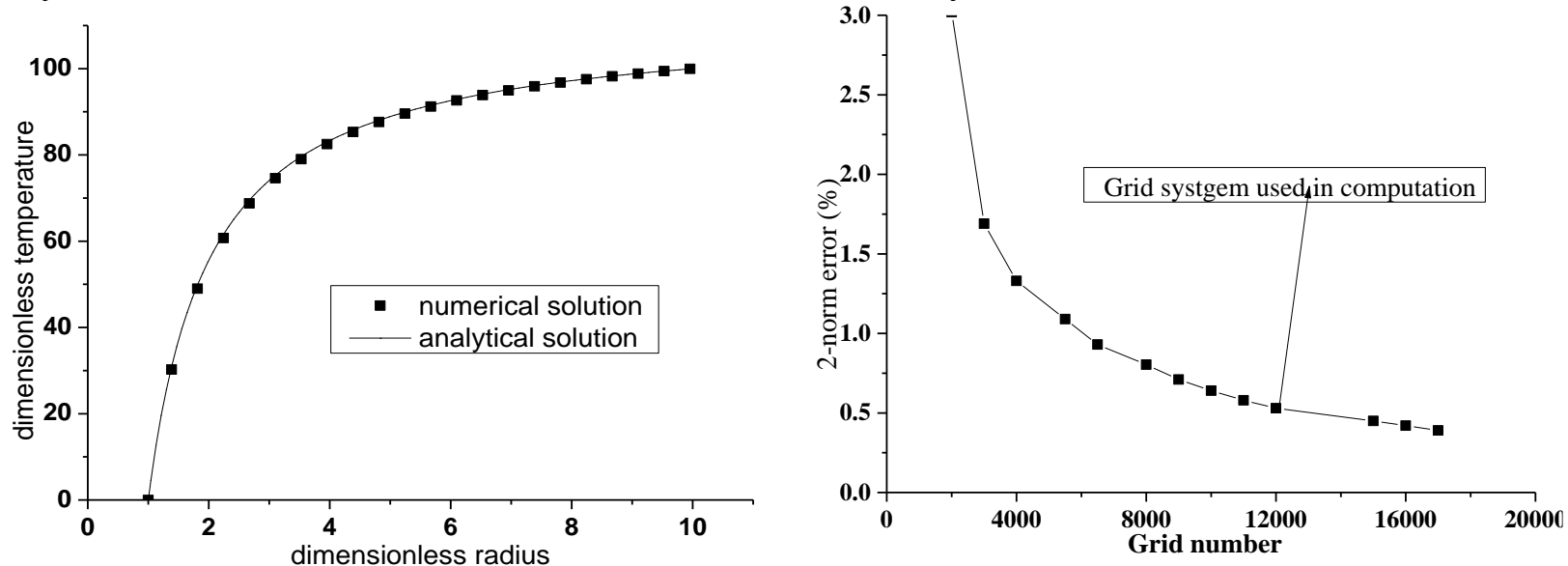

Fig.5 Analytical solution and numerical solution Fig.6 Grids number and error relations

Based on the error obtained from grid-independent solution, the relation among the equation error and grid number is shown in Figure 6. When the grid number reaching 12000, the calculation error control is less than 1\%; with the encryption of the grid, the calculation error is reduced gradually. The analytical solution error is $0.5 \%$, the choice of computational grid number is $47 \times 20 \times 12=11280$.

\section{Conclusion}

According to the three-dimensional equation of heat conduction of the cylinder and sphere, this paper derived the high-accuracy computational discrete scheme with the finite volume method. In the process of the discrete sphere, this paper uses the integral mean value theorem reasonably, which has derived the complex diffusion term more strictly. The new three-dimensional cylindrical coordinates and spherical coordinates provide numerical calculation discrete scheme, which will provide a good reference for the researchers. The application of numerical calculation discrete scheme is written in FORTRAN computer language, which is of stable operation and simple application. This paper have contrasted and analyzed the error, which was controlled within $0.5 \%$. All the work has provided a high-precision numerical calculation discrete scheme for teaching and scientific research.

\section{Acknowledgements}

The work was supported by the youth national natural science fund project of China No. (51504146; 51206101) and the Dr Funds of Shandong Academy of Sciences No. (2015QN023).

I wish to acknowledge the help of Xiaoling SUN and Zunwei LI in Correction translation errors and Mr. Xu provides a lot of help. 


\section{References}

[1]. W. Q. Tao. Numerical Heat Transfer, 2nd ed., Xi'an Jiaotong University Press, China, 2001.

[2]. P. Ding, W. Q. Tao, "Reduced order modeling of fluid flow and heat transfer in tube-fin heat exchanger", Journal of China University of Petroleum (Edition of Natural Science), vol. 35, no. 2, pp. 137-140, 2011.

[3]. S.B. Huang, Z.M. Li, "Numerical analysis of laminar pulsating flow for power law fluid in concentric annulus", Journal of China University of Petroleum (Edition of Natural Science), vol. 36, no 2, pp. 127-136, 2011.

[4]. Y. H. Jin, Q. Guang, Q. Y. Cao, et a.1, "Numerical simulation of gas-phase flow field in vortex finder of cyclone separators", Journal of China University of Petroleum (Edition of Natural Science),vol. 32, no. 6, pp. 109-112, 2008.

[5]. P, Ding, X. Wu, Y. He, et a.1, "A fast and efficient method for predicting fluid flow and heat transfer problems", ASM E Journal of H eat Transfer, vol. 130, no. 7, pp. 1-17, 2008.

[6]. X. C. Deng, "Explore the establishment of thermal conductivity in the spherical coordinate system of differential equations", An Hui Inst Technology, vol. 6, no. 4, pp.58-70, 1987.

[7]. PATANKA SV, SPALDING DB, "A calculation procedure for heat, mass and momentum transfer in three-dimensional parabolic flow”, International Journal Heat Mass Transfer, vol. 15, pp. 1787-1806, 1972.

[8]. Patankar, S. V., "Numerical Heat Transfer and Fluid Flow”, McGraw-Hill, New York, 1980.

[9]. D. K. Tong, S. T. Zhou, A. D. Wu, L. H. Zhang, “Computational Method," Computational Method, University of Petroleum Press, 2004.

[10]. M. H. Xu, Y. B. Zhang, W. Q. TAO, "A modified Delaunay mesh generation method for 2-D domain”, Journal of the University of Petroleum, China( Edition of Natural Science), vol. 25, no. 2, pp.100-105, 2001.

[11]. LEE C K, HOBBS R E. “Automatic adaptive finite element mesh generation over arbitrary two-dimensional domain using advancing front technique", Computers\& Structure, vol. 71, pp.9-34, 1999. 